\title{
Nexos e reflexos da adaptação do enfermeiro à cultura organizacional
}

Nexus and reflections of the nurse's adaptation to organizational culture

Nexos y reflejos de la adaptación del enfermero a la cultura organizacional

\section{Cristiano Pinto dos Santos I, Giovana Calcagno Gomes II, Wilson Danilo Lunardi Filho ${ }^{\text {III }}$ Ariane da Cruz Guedes ${ }^{\mathrm{IV}}$, Adriane Maria Neto de Oliveira ${ }^{\mathrm{v}}$, Ivanete Santiago da Silva Strefling ${ }^{\mathrm{VI}}$}

\begin{abstract}
Resumo: Objetivo: compreender os nexos e reflexos da adaptação do enfermeiro à cultura organizacional do cuidado de enfermagem no âmbito hospitalar. Método: pesquisa qualitativa, exploratório-descritiva, cujos dados foram coletados por meio de entrevistas semiestruturadas com 12 enfermeiros. Para a análise dos dados, foi escolhido o método de análise de conteúdo, por envolver e apreender aspectos subjetivos, emergindo duas categorias: a normalização do cuidado e sobrepujando a adaptação normalizadora. Resultados: o enfermeiro, ao disponibilizar o cuidado, permite que a cultura da organização faça um agenciamento sobre si, sendo esta adaptação otimizada pela falta de exercício da autonomia pelos enfermeiros e por uma identidade singular pouco consolidada. Considerações finais: o reflexo da adaptação à cultura organizacional faz com que o cuidado disponibilizado se fragilize, fomentando de forma insuficiente a proatividade do enfermeiro. Um maior tempo de trabalho favorece a produção de subjetividade singular.
\end{abstract}

DESCRITORES: Cultura organizacional; Adaptação; Enfermeiros; Cuidados de enfermagem

Abstract: Aim: to understand the nexus and reflexes of nurses' adaptation to the organizational culture of nursing care in the hospital setting. Method: qualitative, exploratory-descriptive research, whose data were collected through semi-structured interviews with 12 nurses. For data analysis, the content analysis method was chosen, since it involves and apprehends subjective aspects, emerging two categories: the normalization of care and overcoming the normalizing adaptation. Results: the nurse, by providing the care, allows the organization's culture to make an agency on itself, being this adaptation optimized by the lack of exercise of the autonomy by the

\footnotetext{
I Enfermeiro, Doutor em Enfermagem, Professor da Universidade da Região da Campanha - Urcamp. Bagé, Rio Grande do Sul, Brasil, enfcristiano.ps@hotmail.com https://orcid.org/0000-0001-8298-065

${ }^{\text {II }}$ Enfermeira, Doutora em Enfermagem, Professora Associada da Escola De Enfermagem da Universidade Federal do Rio Grande - FURG. Rio Grande, Rio Grande do Sul, Brasil, giovanagomes@furg.br https://orcid.org/0000-0002-2464-1537

III Enfermeiro, Doutor em Enfermagem, Professor Associado IV, aposentado da Escola De Enfermagem da Universidade Federal do Rio Grande - FURG. Rio Grande, Rio Grande do Sul, Brasil, lunardifilho@terra.com.br https://orcid.org/0000-0002-4633-7632

IV Enfermeira, Doutora em Enfermagem, Professora da Universidade Federal de Pelotas - UFPel. Pelotas, Rio Grande do Sul, Brasil, arianecguedes@gmail.com https://orcid.org/0000-0002-5269-787X

v Enfermeira, Doutora em Enfermagem, Professora Associada da Escola De Enfermagem da Universidade Federal do Rio Grande - FURG. Rio Grande, Rio Grande do Sul, Brasil, adrianeoliveira@furg.br https://orcid.org/0000-0001-9422-423X

VI Enfermeira, Doutora em Enfermagem, Professora da Faculdade Anhanguera. Pelotas, Rio Grande do Sul, Brasil, ivanete25@gmail.com https://orcid.org/0000-0002-5019-6123
} 
nurses and by a singular identity little consolidated. Final considerations: the reflex of the adaptation to the organizational culture makes the available care fragile, insufficiently promoting the nurse's proactivity. A longer working time favors the production of singular subjectivity.

Descriptors: Organizational Culture; Adaptation; Nurses; Nursing care

Resumen: Objetivo: comprender los nexos y reflejos de la adaptación del enfermero a la cultura organizacional del cuidado de enfermería en el ámbito hospitalario. Método: investigación cualitativa, exploratorio-descriptiva; los datos fueron recolectados por medio de entrevistas semiestructuradas con 12 enfermeros. Para el análisis de los datos fue elegido el método de análisis de contenido, por involucrar y aprehender aspectos subjetivos, y del cual emergió dos categorías: la normalización del cuidado y sobreponiendo la adaptación normalizadora. Resultados: el enfermero, al desarrollar el cuidado, permite que la cultura de la organización haga un agenciamiento sobre sí, siendo esta adaptación optimizada por la falta de ejercicio de la autonomía por los enfermeros y por una identidad poco consolidada. Consideraciones finales: el reflejo de la adaptación a la cultura organizacional fragiliza el cuidado disponible, fomentando de forma insuficiente la proactividad del enfermero. Un tiempo más largo de trabajo favorece la producción de subjetividad singular.

Descriptores: Cultura organizacional; Adaptación; enfermeras; Cuidados de enfermería

\section{Introdução}

$\mathrm{Na}$ história do ser humano, os movimentos de adaptação sempre estiveram presentes para atender às exigências do meio mas, também, para acompanhar as evoluções que foram surgindo e que iam exigindo de si demandas diversas. A adaptação pode ser vista como uma resposta permanente àquilo que pode ameaçar o equilíbrio de cada ser humano, uma vez que a exigência, que se impõe ao desenvolvimento de respostas adaptativas para a consecução de determinados objetivos, emerge de forma premente. ${ }^{1}$

O enfermeiro ao realizar os cuidados, seja direta ou indiretamente, assume contornos específicos desenhados pela cultura organizacional estabelecendo, assim, uma conduta coletiva que referencia e norteia os comportamentos e atitudes. Esta acomodação dos enfermeiros à cultura organizacional, concebida por outrem e incorporada por eles, na maioria das vezes sem contestação, cortina uma estruturação organizacional ancorada em crenças e valores estabelecidos e vigentes em um determinado contexto sócio-histórico-cultural, podendo ser relacionada com processos de submissão ao instituído. ${ }^{2}$

Nesse contexto, os enfermeiros vêm desenvolvendo e expressando individualidades norteadas por padronização de normas e condutas já instituídas. Pode, portanto, estar ocorrendo nestas instituições um processo de (des)construção do enfermeiro como profissional, ou seja, ao que parece, os enfermeiros, geralmente, colocam em segundo plano seus conhecimentos e concepções, passando a ter o trabalho delineado pelos aspectos que permeiam a cultura organizacional dos respectivos ambientes de trabalho dos locais em que atuam. 
Cabe resgatar que, por meio da produção de bens e valores que constroem a sociedade, o trabalhador realiza uma auto identificação social e se apresenta a ela, produzindo assim, não só objetos, mas uma condição que é particularmente sua. Dessa forma, por representar esta trajetória, o significado do trabalho do enfermeiro de cuidar precisa transcender a estrutura socioeconômica, a cultura e os valores, incorporando sua produção de subjetividade, como sujeito proativo. ${ }^{3}$

Percebendo a inter-relação existente entre a adaptação à cultura organizacional e o cuidado disponibilizado pelo enfermeiro aos pacientes, este estudo teve como questão: quais os nexos e reflexos da adaptação do enfermeiro à cultura organizacional no cuidado de enfermagem disponibilizado? Desse modo, teve como objetivo: compreender os nexos e reflexos da adaptação do enfermeiro à cultura organizacional no cuidado de enfermagem no âmbito hospitalar.

\section{Método}

Trata-se de um estudo de cunho qualitativo, exploratório-descritivo em que foram enfocados aspectos não mensuráveis da vida das pessoas, que implicam comportamentos adaptáveis e normativos. Optou-se pelo trabalho de campo, pois, a realização de uma pesquisa qualitativa pressupõe interação entre o pesquisador e os participantes, almejando alcançar os resultados esperados. ${ }^{4}$

A coleta de dados ocorreu no contexto de um hospital do município de Bagé (Rio Grande do Sul) nos meses de outubro e novembro de 2015 por meio de entrevista semiestruturada individual. Foram incluídos na pesquisa 12 enfermeiros, de um total de 25, vinculados a essa instituição, conforme Consolidação das Leis do Trabalho (CLT).

As entrevistas foram realizadas em uma sala de reuniões para manter a privacidade dos participantes e foram abordadas questões sobre o processo de trabalho do enfermeiro. Tiveram duração média de 30 minutos, sendo audiogravadas e transcritas na íntegra posteriormente. $\mathrm{O}$ número de participantes foi determinado pela saturação dos dados.

Posteriormente, os dados foram analisados utilizando a técnica de análise de conteúdo, ${ }^{5}$ a qual inicialmente foi realizada uma leitura flutuante para a sistematização dos dados e, após, identificou-se nas falas, as unidades de significado para a compreensão do depoimento. Por fim os dados foram tratados agrupando as unidades de significado, de acordo com suas semelhanças. 
Todos os preceitos éticos foram seguidos de acordo com a resolução 466/12, tendo sido a pesquisa aprovada por um Comitê de Ética em Pesquisa da Universidade Federal do Rio Grande sob parecer no 161/2015 (CAAE: 46809715.8.0000.5324). Os participantes assinaram o Termo de Consentimento Livre e Esclarecido em duas vias antes das entrevistas, sendo identificados pela letra "E” seguida do número sequencial das entrevistas.

\section{Resultados e discussões}

Em relação às características do grupo de participantes que fez parte deste estudo, destaca-se a participação de 12 enfermeiros, representados por oito mulheres e quatro homens, com idades que variavam entre 23 e 49 anos. O tempo de formação variou entre 3 e 15 anos e todos realizaram curso de pós-graduação (especialização), em diferentes áreas. No entanto, nenhum dos participantes cursa ou cursou especialização ou mestrado voltado para o eixo gerencial/organizacional. O tempo de trabalho na instituição variou de 1 a 14 anos.

Percebe-se que entre os participantes existe a predominância do sexo feminino $(66,66 \%)$. No entanto, essa particularidade somada a idade e o tempo de formação não foram determinantes para a adaptação à cultura organizacional, pois o estudo demonstrou que o tempo de atuação no local de trabalho é o aspecto que mais contribui para o estabelecimento da normalização. Talvez um empoderamento maior do enfermeiro sobre as questões organizacionais, por meio de especialização ou mestrado em gestão, possibilitasse uma modificação no cenário explorado, visto que o conhecimento e reflexão sobre as práticas laborais são fundamentais para a reorganização do processo de trabalho.

\section{A normalização do cuidado}

O enfermeiro tem como essência da sua atuação profissional o cuidado junto às pessoas e, especialmente na esfera hospitalar, atua de forma autêntica, na produção de sentido, em meio ao processo de hospitalização o qual, muitas vezes, produz sentimentos de dor, perdas e limitações. Como profissional, tem a capacidade de observar agravos de saúde, sejam biológicos, sociais, psíquicos ou espirituais, pois a essência da sua profissão é perceber a pessoa como um ser integral e interativo. ${ }^{6}$

No entanto, para que possa desenvolver suas atividades de forma plena e de acordo com seus princípios éticos e morais, torna-se fundamental que assuma e se aproprie de sua identidade, cultivando e exercendo sua autonomia para que, assim, possa fugir de alguns padrões de conduta pré-estabelecidos e transcender a assistência modulada, aumentando sua capacidade de autodeterminação. ${ }^{7}$ Alguns participantes relataram que não conseguem ser quem 
realmente gostariam e que o exercício de maior autonomia poderia enriquecer o processos de trabalho para, repercutindo de forma mais resolutiva e proativa no cuidado disponibilizado, favorecendo a recuperação do paciente.

Quanto mais autonomia o enfermeiro tem, melhor é o cuidado disponibilizado. [...] Se tivéssemos mais autonomia, com certeza, o paciente ia ser favorecido. [...] É difícil fazer a diferença, fazendo o que os outros querem. (E5)

O enfermeiro tem pouca autonomia, está sempre fazendo coisas que não acredita que teria que fazer. [...] Assim fica difícil dar o melhor de ti. (E2)

Acho que a autonomia ajuda o enfermeiro a fazer as coisas da melhor forma. [...] Quando determinada pessoa não autoriza ou não aceita o que tu fazes, já corta teu serviço, tua assistência. (E12)

Eu sempre me queixo muito da falta de autonomia do enfermeiro. [...] Eu acho que o enfermeiro, para fazer um bom serviço, desempenhar um bom serviço, seja na supervisão ou como enfermeiro de unidade, eu digo a nível hospitalar, tem que ter autonomia. (E11)

Existe uma estreita relação entre a produção de subjetividade autêntica e a autonomia concedida ou conquistada. A adaptação à cultura organizacional pode ter como uma das diretrizes a falta de exercício da autonomia pelos enfermeiros, pois, no momento em que esta não é exercida e também não é desejo fundamental, o profissional passa a (re)produzir as práticas pré-estabelecidas, internalizando a subjetividade produzida por outrem, tonando-se um pilar irremovível. ${ }^{8} \mathrm{O}$ nexo causal desta adaptação pode ser o auto enquadramento à cultura organizacional, que pode ser consciente ou inconsciente, pois o cortinamento da subjetividade singular nem sempre é intencional ou voluntário.

A autonomia do enfermeiro é um dos fatores fundamentais para que possa realizar um trabalho diferenciado mas, para isso, ele precisa ter a coragem de se assumir como profissional, mostrando sua competência, responsabilidade e sua forma única de trabalhar. ${ }^{9}$ Contrapondo-se a estes relatos, houve referência que a autonomia não é um fator importante e diferencial no trabalho do enfermeiro, destacando que o importante é ter vontade e amor ao trabalho.

Eu acho que a autonomia não ajuda em nada o enfermeiro. Se ele tiver boa vontade, isso é que vai fazer a diferença.(E3) 
Cabe ressaltar que esse relato parece ser condizente com a postura profissional daqueles que difundem a cultura da organização aos demais e faz com que se adaptem, incondicionalmente, às normas estabelecidas. Talvez, por assumir uma identidade institucional e não pessoal, o enfermeiro não visualize o exercício da autonomia profissional como fator essencial no trabalho trazendo reflexos negativos para sua profissão, pois passa a ser somente mais uma engrenagem no processo de trabalho.

Desse modo, mostra-se importante discutir sobre a autonomia profissional dos enfermeiros, não como mero interesse corporativo e privado, mas como possibilidade de afirmação de suas identidades, como profissionais ativos e indispensáveis na construção de serviços de saúde mais competentes, solidários e humanizados. ${ }^{10}$ Ao discutirmos em uma perspectiva de construção e consolidação de sua identidade, o enfermeiro pode passar a incorporar a missão de sujeito ativo, proativo e reacionário, ou seja, pode desmobilizar a massificação de subjetividade envolvida em seu trabalho. Dessa forma, pode contornar os reflexos normalizadores da cultura organizacional possibilitando uma nova forma de cuidar.

A normalização subjetiva implica uma identidade marcada pela significação que o sujeito tem de si mesmo e dos demais colegas enfermeiros. Cada cultura possui uma maneira de delinear as identidades, ${ }^{11}$ mas, os relatos revelam que as idiossincrasias, filosofias, valores e ideias dos enfermeiros são tomadas por um sistema modular que reduz tudo a um confinamento estrutural padrão, fazendo com que não consigam ser eles mesmos em seu trabalho, produzindo um reflexo de pouco dinamismo, assumindo uma identidade que não condiz com aquela construída, durante sua formação. Os enfermeiros anseiam por uma identidade profissional sólida, com a qual tenham liberdade e poder de tomada de decisão sobre questões específicas de sua área de competência, sem manipulação de seu fazer. ${ }^{12}$

Dessa forma, o cuidado disponibilizado pelo enfermeiro segue um itinerário que tem como reflexo uma identidade cartesianamente codificada e, com isso, novas formas de discutir, refletir e implementar práticas são aproximadas de um abismo, em que a vontade de suplantar esse limite pode ser o início de um fim. Os relatos destacam que os enfermeiros não conseguem serem eles mesmos durante as atividades laborais, principalmente logo após serem contratados, assumindo uma identidade programada pela cultura organizacional. Com isso, o cuidado disponibilizado passa a ser condicionado e pouco evolutivo.

Não dá para seres tu mesmo no trabalho, porque parece que tu feres os princípios de fulano, enfim. [...] É uma coisa entranha. Eu sempre achei isso tudo muito estranho. [...] Quem perde com isso, é o paciente. (E5) 
Eu não consigo ser eu mesma no trabalho [...] Aquele que se nega, que faz cara feia, aquele ali não é lembrado. [...] Não tem como ter o teu próprio estilo de trabalho e fazer a diferença. (E4)

Nem tudo tu podes falar, nem tudo tu podes te meter, então, a gente deixa de ser, de ter aquela autenticidade que a gente tinha, quando a gente entrou na Universidade, para ser um membro transformador, criador [...] Com isso, a assistência fica prejudicada. (E9)

Os enfermeiros, muitas vezes, têm como reflexos da adaptação à cultura organizacional, dificuldades em desfragmentar sua identidade pois, caso ultrapassem esta barreira, deixarão de ser membro institucional modelo, fugindo do padrão normal construído culturalmente pela organização hospitalar, confirmando que a identidade é construída, a partir de padrões oferecidos pela estrutura social. ${ }^{13}$ Em outras palavras, a pessoa desenvolve sua identidade, por meio de articulações entre igualdades e diferenças, que favorecem que ela promova uma consciência de si, por meio de comparações que faz nas relações sociais que mantém. ${ }^{14}$

Percebe-se que a consolidação da identidade relaciona-se com a produção de subjetividade singular. Quanto menos consolidada a identidade estiver, menor a subjetividade singular produzida pois, ao deixar a essência do eu em segundo plano, o enfermeiro assume uma identidade transitória que é um produto adaptativo facilmente influenciável pelos fatores da cultura organizacional. Essa reflexo da adaptação à cultura organizacional faz com que o cuidado disponibilizado se fragilize, pois novas práticas deixam de ser discutidas e analisadas para serem implementadas.

\section{Sobrepujando a adaptação normalizadora}

Sobrepujar a adaptação normalizadora estimulada pela cultura organizacional, não é uma tarefa descomplicada, pois as forças institucionais e serializadoras atuam de forma sinérgica, dificultando a produção e articulação de ações que possam abalar suas estruturas. No entanto, por meio dos relatos, salienta-se que enfermeiros mais antigos destacam que depois de certo tempo de trabalho, a identidade é singularmente fortalecida, possibilitando que eles possam adquirir uma personalidade mais próxima daquela que planejaram durante sua formação.

Dessa forma, passam a exercer o poder de determinar alguns aspectos organizacionais e assistenciais, começando a ter possibilidades de produzir uma subjetividade autêntica e transformadora, mesmo que essa possa impactar contra os interesses massificadores da 
Nexos e reflexos da adaptação do enfermeiro à cultura organizacional I 8

instituição. Com isso, o cuidado passa a ser mais contextualizado e resolutivo, além de ser mais permeável às mudanças.

Agora, devido ao tempo que tenho, acho que posso ser mais eu no trabalho e determinar algumas coisas a serem feitas. [...] A instituição não vê isso, mas os pacientes sim. (E3)

Eu vejo que o enfermeiro, já com um certo tempo, como eu assim, a gente consegue ser mais autêntico e fazer fluir melhor as coisas e a gente acaba ajudando mais os pacientes. (E6)

Quando a gente entra na instituição, não é fácil, ainda mais sendo nova. [...] Acho que conseguimos mostrar quem realmente somos e nosso bom trabalho para o paciente, somente após um bom tempo de casa. (E7)

O enfermeiro até ter autoconfiança e iniciativa para mudar algumas coisas no seu trabalho ou para o paciente, passa por um processo lento, que pode durar de meses a anos. (E8)

Existe uma perspectiva de mudança no perfil dos enfermeiros dentro da unidade hospitalar, com o passar do tempo. Depois de alguns anos de trabalho, os enfermeiros começam a assumir um papel guiado por um novo olhar das competências e habilidades fundamentais para que possam promover uma renovação nas práticas desenvolvidas e, assim, indicarem novos caminhos revestidos de autenticidade. ${ }^{15}$ O enfermeiro, após certo tempo de trabalho na instituição hospitalar, passa a ter uma percepção diferente da importância de produzir uma subjetividade legítima, vendo este processo como uma evolução de sua força produtiva, em sua forma mais desenvolvida. Pode-se perceber esse fenômeno como um "efeito rebote" do tempo de normalização serializadora que o enfermeiro vivenciou.

Dessa maneira, passa a unificar suas concepções, revolucionando sua própria produção de trabalho, utilizando os conhecimentos e equipamentos de modo particular e diferenciado. No entanto, essa nova subjetividade construída parece estar alicerçada em uma rocha revestida de verdade e razão absoluta, fazendo com que os demais enfermeiros tenham que congregar essas concepções sem poder demonstrar todo o seu potencial inovador e subjetivista. ${ }^{16}$ Parece que o ciclo de normalização à cultura organizacional é um reflexo que se retroalimenta no ambiente hospitalar, sendo possível perceber um enfermeiro normalizando e outro sendo normalizado de forma contínua. 
É preciso que os enfermeiros se concentrem em construir uma capacidade de gerenciar de forma mais horizontal, aceitando a comunicação aberta, a culpa, o trabalho em equipe e o conhecimento contínuo da cultura organizacional. ${ }^{17}$ Caso alguns enfermeiros apresentem certa resistência para submergir nesse universo subjetivo de propriedade alheia, podem ter que experimentar mudanças em sua rotina de trabalho, como mudar de unidade ou de turno de trabalho. Cabe ressaltar que os vínculos firmados do enfermeiro com determinado espaço se tornam fator importante para a disponibilização de um cuidado especializado e resolutivo, pois, quanto maior for o conhecimento do local, maiores serão as possibilidades de uma boa análise situacional do ambiente, identificando pontos fortes e fracos, além de favorecer um planejamento prospectivo. ${ }^{18}$

Partindo dessa explanação, entende-se que um enfermeiro que conheça e domine com excelência seu ambiente de trabalho, pode e deve disponibilizar um cuidado especializado, resolutivo e integral a quem está na ponta do processo, ou seja, o paciente que recebe, de forma direta ou indireta, os produtos gerados no seu processo adaptativo. Percebe-se, por meio das falas, que alguns enfermeiros são remanejados de seu local de trabalho ao questionar ou contrariar alguns comandos estabelecidos por enfermeiros mais antigos ou sofrem com ameaças de demissão.

Eu até tentei mudar algumas coisas, mas não consegui dar continuidade, pois, depois de um mês, me trocaram de unidade. [...] Isso é ruim não só para nós, mas para o paciente também. (E4)

Se tu tentas implementar uma rotina, é complicado. [...] Se tu insistes, o desfecho é a mudança de unidade. [...] Aí, o que tu planejaste, pensando no paciente, vai por água abaixo. (E1)

Já quiseram me despedir. [...] Pelo pensamento, não por questões de serviço. [...] Não fui para a rua, mas me mudaram de unidade. (E10)

As questões discutidas ficam evidentes com o relato de um enfermeiro normalizador sobre possíveis trocas de unidade.

Eu tento fazer com que as coisas sejam bem desenvolvidas e executadas. [...] Se tem algum problema, eu tento resolver, e, se não der, solicito o remanejamento. (E6) 
No trabalho do enfermeiro, é comum alguns interesses se desencontrarem, ocorrendo uma desorganização temporária nas atividades, repercutindo no aumento do estresse, sobrecarga de trabalho e rotatividade dos profissionais. ${ }^{19} \mathrm{Nem}$ sempre o enfermeiro consegue assumir sua singularidade com consistência, pois se choca com outros enfermeiros mais subjetivados, do ponto de vista de produção e reprodução de sentidos.

O nexo para assumir essa postura pode ser o medo de ser demitido, pois a pessoa quando é submetida à ameaça de demissão consente com aspectos que discorda, pois o medo é, sobretudo, uma vivência subjetiva que gera repercussões psicológicas. ${ }^{20}$ Dessa forma, o cuidado disponibilizado fica limitado à forma como foi pré-programado, estanque frente a um universo dinâmico e rapidamente mutante, que é a atenção à saúde, em especial, a atenção à saúde em âmbito hospitalar, desfavorecendo, assim, seu bem maior que é o paciente.

A Enfermagem realiza suas atividades adentrando nos subsistemas de produção que fazem parte de um cuidado complexo, sendo influenciada pelas características organizacionais e, essa por sua vez, esquematiza o produto gerado, ou seja, gera reflexos na qualidade do cuidado disponibilizado. ${ }^{21}$

Espera-se que o Enfermeiro possa fazer da adaptação uma maneira de crescimento profissional para que, desta forma, elabore ferramentas capazes de promover um cuidado efetivo e que atenda às necessidades de saúde de seus pacientes. Para isso, terá que deixar sua inibição e omissão de lado, criando mecanismos de defesa contra os nexos que o fazem se adaptar à cultura organizacional, sem interiorizar os comandos dos demais como verdades inquestionáveis

\section{Considerações finais}

O enfermeiro, ao disponibilizar o cuidado, permite que a cultura da organização faça um agenciamento sobre si, seguindo os comandos sem pensar muito no valor de seus atos. Este cuidado segue um itinerário que tem como guia uma identidade cartesianamente codificada e, com isso, novas formas de discutir, refletir e implementar práticas são aproximadas de um abismo, onde a vontade de suplantar esse limite pode ser o início de um fim.

A adaptação à cultura organizacional é otimizada pela falta de exercício da autonomia pelos enfermeiros, mas também por uma identidade singular pouco consolidada, assim como pelo medo de demissão. Outro fator que emergiu de forma intensa foi o fato de enfermeiros mais antigos utilizarem punições, como a troca de unidade para forçar uma normalização dos demais, fazendo assim com que o cuidado seja cartesiano e pouco evolutivo. 
No entanto, alguns participantes relataram que após certo tempo de vínculo institucional, os enfermeiros passam a ter o poder de determinar alguns aspectos organizacionais e assistenciais, começando a ter possibilidades de produzir uma subjetividade autêntica e transformadora, mesmo que esta impacte contra os interesses massificadores da instituição. Com isso, o cuidado passa a ser mais contextualizado e resolutivo, além de mais permeável às mudanças.

O estudo favorece a prática de enfermagem uma vez que promove reflexões e faz emergir questões peculiares ao trabalho. No entanto, há uma limitação no estudo em função de ter sido desenvolvido em apenas um Hospital e com enfermeiros com o mesmo regime de trabalho, sendo todos balizados pela CLT. Além disso, torna-se imperativo o desenvolvimento de mais estudos que aproximem e apreendam as relações estabelecidas na adaptação do enfermeiro à cultura organizacional.

O nexo causal desta adaptação pode ser o auto enquadramento à cultura organizacional, que pode ser consciente ou inconsciente, pois o cortinamento da subjetividade singular nem sempre é intencional ou voluntário. No entanto, o medo da demissão parece ser o nexo mais acentuado para que ocorra a normalização serializadora.

O reflexo da adaptação à cultura organizacional faz com que o cuidado disponibilizado se fragilize, fomentando de forma insuficiente a proatividade do enfermeiro. $O$ ciclo de normalização é um reflexo que se retroalimenta, onde um enfermeiro normaliza e outro é normalizado de forma sucessiva.

\section{Referências}

1. Gonçalves FGA, Leite GFP, Souza NVDO, Santos DM. O modelo neoliberal e suas repercussões para o trabalho e para o trabalhador de enfermagem. Rev Enferm UFPE Online [Internet]. 2013 nov [acesso em 2017 dez 10];7(11):6352-9. Disponível em: https://periodicos.ufpe.br/revistas/revistaenfermagem/article/viewFile/12279/14933 doi: 10.5205/reuol.3794-32322-1-ED.0711201306

2. Hahtela N, McCormack B, Paavilainen E, Slater P, Helminen M, Suominen T. The relationship of workplace culture with nursing-sensitive organizational factors. J Nurs Adm [Internet]. 2015 jul-ago [acesso em 2018 set 01];47(7-8):370-6. Disponível em: https://www.ncbi.nlm.nih.gov/pubmed/26204378 doi: 10.1097/NNA.0000000000000217

3. Souza DO, Gonçalves FGA, Pires AS, David HMSL, Valéria N. Influência do neoliberalismo na organização e processo de trabalho hospitalar de enfermagem. Rev Bras Enferm [Internet]. 2017 jan 
[acesso em 2018 set 01];70(5):961-9. Disponível em: http://www.scielo.br/pdf/reben/v70n5/pt_0034-7167reben-70-05-0912.pdf doi: http://dx.doi.org/10.1590/0034-7167-2016-0092

4. Minayo MCS. O desafio do conhecimento: pesquisa qualitativa em saúde. São Paulo: Hucitec; 2013.

5. Bardin L. Análise de conteúdo: Laurence Bardin. São Paulo: Edições 70; 2015.

6. Kinchen E. Development of a quantitative measure of holistic nursing care. J Holist Nurs [Internet]. 2015 set [acesso em 2017 dez 08];33(3):238-46. Disponível em: https://www.ncbi.nlm.nih.gov/pubmed/25536967 doi: 10.1177/0898010114563312

7. Souza GJ, Paula MAB. Construção da identidade do enfermeiro: revisão integrativa da literatura. Rev Rede Cuid Saúde [Internet]. 2016 jun [acesso em 2017 dec 13];10(1):1-17. Disponível em: http://publicacoes.unigranrio.edu.br/index.php/rcs/article/view/2727

8. Ramos AM, Barlem ELD, Barlem JGT, Rocha LP, Dalmolin GL, Figueira AB. Adaptação cultural e validação da Moral Distress Scale Revised para enfermeiros. Rev Bras Enferm [Internet]. 2017 mar [acesso em 2018 set 01];70(5):1063-70. Disponível em: http://www.scielo.br/ scielo.php?script=sci_arttext\&pid=S0034-71672017000501011\&lng=en\&nrm=iso\&tlng=pt doi: http://dx.doi.org/10.1590/0034-7167-2016-0518

9. Avila LI, Silveira RS, Lunardi VL, Fernandes GFM, Mancia JR, Silveira JT. Implicações da visibilidade da enfermagem no exercício profissional. Rev Gaúch Enferm. 2013;34(3):102-109.

10. Pereira MS. Lei do exercício profissional de enfermagem e a autonomia profissional do enfermeiro. Enferm Foco (Brasília) [Internet]. 2015 jun [acesso em 2017 dez 08];4(3/4):171-4. Disponível em: http://revista.cofen.gov.br/index.php/enfermagem/article/view/543/226

11. Ettinger VMM, Jesus Júnior G, Setenta AM, Cavalcante AL. Cultura, identidade e gênero: tecendo a rede de mulheres de comunidades extrativistas e pesqueiras do sul da Bahia. Rev Interdiscip Gest Soc [Internet]. 2016 set [acesso em 2017 dez 01];4(3):151-9. Disponível em: https://portalseer.ufba.br/index.php/rigs/article/view/10188/11562 doi: http://dx.doi.org/10.9771/23172428rigs.v4i3.10188

12. Umpiérrez AHF, Merighi MAB, Muñoz LA. Percepções e expectativas dos enfermeiros

sobre sua atuação profissional. Acta Paul Enferm [Internet]. 2013 [acesso em 2017 dez 08];6(2):165-71. Disponível em: http://www.scielo.br/pdf/ape/v26n2/v26n2a10.pdf doi: http://dx.doi.org/10.1590/S010321002013000200010

13. Hoeve Y, Jansen G, Roodbol P. The nursing profession: public image, self-concept and professional identity. A discussion paper. J Adv Nurs [Internet]. 2013 [acesso em 2017 dez 05];70(2):295-309. Disponível em: https://www.ncbi.nlm.nih.gov/pubmed/23711235 doi: 10.1111/jan.12177 
14. Lima MM, Reibnitz KS, Prado ML, Kloh D. Integralidade como princípio pedagógico na formação do enfermeiro. Texto \& Contexto Enferm [Internet]. 2013 [acesso em 2017 dez 09]; 22(1):106-13. Disponível em: http://www.scielo.br/pdf/tce/v22n1/pt_13.pdf doi: http://dx.doi.org/10.1590/S0104-07072013000100013

15. Sebold LF, Carraro TE. Autenticidade do ser-enfermeiro-professor no ensino do cuidado de enfermagem: uma hermenêutica heideggeriana. Texto \& Contexto Enferm [Internet]. 2013 [acesso em 2017 dez 09];22(1):22-8. Disponível em: http://www.scielo.br/pdf/tce/v22n1/pt_03.pdf doi: http://dx.doi.org/10.1590/S0104-07072013000100003

16. Guattari F, Rolnik S. Micropolítica cartografias do desejo. Petrópolis (RJ): Vozes; 2010.

17. Ammouri AA, Tailakh AK, Muliira JK, Geethakrishnan R, Al Kindi SN. Patient safety culture among nurses. Int Nurs Rev [Internet]. 2015 [acesso em 2017 dez 10];62(1):102-10. Disponível em: https://www.ncbi.nlm.nih.gov/pubmed/25495946 doi: 10.1111/inr.12159

18. Vaughan L, Slinger T. Building a healthy work environment: a nursing resource team perspective. Nurs Leadersh (Tor Ont). 2013;26(Spec No 2013):70-7.

19. Guse C, Carvalho DR. Rotatividade nos profissionais de enfermagem. Publ UEPG Ciênc Biol Saúde. 2015; 21(1):47-64.

20. Dejours C. A banalização da injustiça social. Rio de Janeiro (RJ): Fundação Getúlio Vargas; 2011.

21. Schwonke CRGB, Lunardi Filho WD, Lunardi GL, Silveira RS, Rodrigues MC, Guerreiro MO. Cultura de segurança: a percepção dos profissionais de enfermagem intensivistas. Enfermería Glob [Internet]. 2016 [acesso em 2017 dez 07];15(1):208-43. Disponível em: http://scielo.isciii.es/pdf/eg/v15n41/pt_administracion3.pdf

\section{Autor correspondente}

Cristiano Pinto dos Santos

E-mail: enfcristiano.ps@hotmail.com

Endereço: Rua Cláudio Manoel da Costa 101, bairro Tiaraju, Bagé, Rio Grande do Sul, Brasil.

CEP: $96422-080$

\section{Contribuições de Autoria}

1 - Cristiano Pinto dos Santos

Planejamento do projeto de pesquisa, análise e interpretação dos dados, redação e revisão crítica. 
2 - Giovana Calcagno Gomes

Análise e interpretação dos dados; revisão crítica.

3 - Wilson Danilo Lunardi Filho

Análise e interpretação dos dados; redação e revisão crítica.

4 - Ariane da Cruz Guedes

Análise e interpretação dos dados

5 - Adriane Maria Neto de Oliveira

Redação e revisão crítica.

Nome 6 - Ivanete Santiago da Silva Strefling

Revisão crítica.

\section{Como citar este artigo}

Santos CP, Gomes GC, Lunardi Filho WD, Guedes AC, Oliveira AM, Strefling ISS. Nexos e reflexos da adaptação do enfermeiro à cultura organizacional. Rev. Enferm. UFSM. 2019 [Acesso em: 2019 jun 15];vol 9 ex:1-17. DOI:https://doi.org/10.5902/2179769230699 